\title{
Phase composition and strength of cement stone with a complex additive
}

\author{
Galina Shabanova ${ }^{1}$, Alla Korohodska $^{1}$, and Vita Shumejko ${ }^{1, *}$ \\ ${ }^{1}$ National Technical University "Kharkov Polytechnic Institute" (NTU "KhPI"), Technology of \\ Ceramics, Refractory Materials, Glass and Enamels Department, Kyrpychova str. 2, 61002, Kharkiv, \\ Ukraine
}

\begin{abstract}
Ecologically friendly construction is impossible without the use of materials that are characterized by a low emission of dangerous substances from the surface and have other appropriate properties. A solution of the problem related to the elaboration of special measures taken to reduce the emission of ammonia from construction materials and the structures manufactured using the Portland cement has been offered. An optimized composite dope was developed and optimized for the Portland cement composition that contains the nitric acid calcium, which is one of the ingredients that can considerably reduce the concentration of nitrogencontaining compounds, making thus the construction materials less dangerous to the human health. Strength characteristics of the cement stone with the composite dope were studied and it was established that a level of the intensification of a gain in the strength is rather significant and by the seventh day the hardening exceeds the values peculiar for the dope-free compositions at an age of 28 days by more than $45 \%$.
\end{abstract}

\section{Introduction}

Technological progress in the field of construction material science requires an increase in the efficient action of the dopes of a different type. The use of chemical dopes is one of the most universal and available methods of the control of concrete technology and adjustment of its properties. In the past some chemical products were widely used as the dopes for construction materials and at the present time the dopes specially prepared for cement solutions and concretes are more preferable. Many dopes that improve certain characteristics of the concrete mixture or the concrete sometimes fail to improve other characteristics and even worsen them. To overcome these side effects we use composite dopes that consist of several independent components. In addition to evident advantages, the chemicalization of concrete production technologies poses new problems related to the quality of concrete from the standpoint of ecology and hygiene [1, 2].

At the present time, a concept of the "green" (or ecological) construction, in particular the construction of such objects that have a minimum negative effect on the environment becomes increasingly popular. The buildings and installations erected according to the ecological concept will provide comfortable and safe living conditions and also preserve

\footnotetext{
*Corresponding author: shum-vita@ukr.net
} 
and improve the environment. Meanwhile, the experts [3] believe that building and finishing materials and structures, including concrete ones that release toxic substances are one of the sources of chemical pollution of the air inside the living quarters. The most important aspect is the problem of ammonia emission from concrete structures in the living quarters of residential and public buildings. The emission of ammonia by the concrete is a widely spread phenomenon peculiar for newly built houses and it has a dreadful effect on human beings and the environment.

\section{Analysis of the latest research papers and publications}

The analysis of scientific sources and patent information allows us to draw a conclusion that the problem of ammonia emission by the concrete is also peculiar for the foreign practice of construction [4-7]. It was noted, that ammonia release sources are Portland cement-based solutions and concretes. The paper [8] gives consideration to one more possible reason for the accumulation of nitrogen-containing compounds in the Portland cement; in particular this is the formation of Portland cement clinker under reduction conditions due to the annealing and cooling. The research done enabled the theoretical substantiation of the possibility of formation of iron nitrides during the formation of Portland cement clinker that can be a source of ammonia emission from construction materials and the structures. A solution was found for the elaboration of special measures taken to reduce the ammonia emission from construction materials and the structures fabricated using the Portland cement. The authors [8] assume that ammonia emission sources accumulated in the hardened cement stone have a continuous harmful effect due to a permanent emission of ammonia, and the research done by them showed a positive influence of the dopes that are capable of intensifying the ammonia emission during the cement mixing and at an early stage of the hardening of cement stone. It was shown that nitric acid calcium added in low concentrations facilitates an early ammonia emission from the cement stone.

\section{Defining research goals and problems}

Ecologically friendly construction is impossible without the use of materials characterized by a low emission of dangerous substances from the surface and that have other appropriate properties. In this connection, a creation of the composite dope for the Portland cement composition that includes $\mathrm{Ca}\left(\mathrm{NO}_{3}\right)_{2}$ is of great interest. The latter is one of the gradients that can considerably reduce the concentration of nitrogen-containing compounds making the construction materials less dangerous to the human health.

Since the Portland cement is an active component of the concrete, the characteristics of this artificial material will be defined to a great extent by the properties of hardened Portland cement stone. The use of nitric acid calcium in substantial concentrations (more than 1 mass \%) is efficient for the control of time required for the hardening of cement stone [9]. It is clear that these factors are not efficient at very low concentrations of nitric acid calcium. However, the research showed that $\mathrm{Ca}\left(\mathrm{NO}_{3}\right)_{2}$ added even in low concentration has a substantial influence on physical and mechanical properties of the cement stone.

The composition of composite dopes should be "designed" to allow the components to intensify the effects provided by each component individually.

An efficient use of the dopes for contemporary construction technologies is defined by the choice of their rational composition taking into consideration the chemical phenomena of additivity and synergism, i.e. a compatibility of the dopes of a different nature during their interaction, which enables the intensification of obtained effects. For cement systems, 
the efficiency is defined by the hydration activity that characterizes an early strength and the grade strength.

The purpose of this scientific paper was to optimize cement-containing compositions with modifying dopes and analyze the phase composition of hydration products that provides for a decrease in the emission of ammonia from construction materials and the structures fabricated using the Portland cement.

\section{Major Research}

When selecting a dope and defining a dose we must clearly see the role of each component in the polyfunctional modifier. All the composite dopes can conventionally be subdivided into the three groups: the mixture of electrolytes; the mixture of surfactants and the mixtures of electrolytes and surfactants. The introduction of electrolytes enables a control of the hardening rate and structural and mechanical properties and the surfactants control the mobility, imparting some special properties, for example rheological to the cement solution and the concrete mixture.

To do research we used the dope-free Portland cement of a PC I-500-H grade (DSTU BV.2.7-46:2010) produced by PJSC "Eurocement-Ukraine". The nitric acid calcium (NC), sodium hexametaphosphate (SHMP) and sodium tripolyphosphate (STPP) were used as modifying dopes.

Sodium salts of phosphoric acids are the electrolytes and perform as the plasticizing additive. The sodium hexametaphosphate has a polymer nature and it is known as the Graham's salt. Therefore the mixture of sodium hexametaphosphate and sodium tripolyphosphate contributes to the properties not only of electrolyte. This mixture forms duel electric layers on hydrated cement particles and contributes to the rarefaction of cement paste due to the electrostatic repulsion. Beside, having the surfactant properties, the mixture adsorbs on cement particles and amplifies the rarefaction-plasticization effect due to their steric repulsion during the cycle rupture and the orientation of linear polymer chains being forming.

Experimental verification of the individual compatibility of tested dopes with Portland cement enabled the establishment of their optimal quantity to be used to provide an improvement in the strength characteristics of cement stone in comparison with those of the produced Portland cement.

To define an optimal composition for the offered composite dope consisting of the SHMP, STPP and NC mixture in different proportions and carry out the comparative tests we fabricated the following cement compositions and analyzed their compression strength:

-1)- PC I-500-H;

-2)- PC I-500-H + $0.075 \%$ (33.33 \% NC : $33.33 \%$ STPP : $33.33 \%$ SHMP);

-3)- PC I-500-H + $0.075 \%$ (33.33\% NC : $44 \%$ STPP : $22.67 \%$ SHMP);

-4)- PC I-500-H +0.075 \% (33.33\% NC : $22.67 \%$ STPP : $44 \%$ SHMP).

To define the strength, the specimens in the form of cubes $20 \times 20 \times 20 \mathrm{~mm}$ were made of the cement paste, A compression strength of the specimens of hydrated dope-free Portland cement and the doped cement were tested using the hydraulic press during their hardening for 2, 7 and 28 days. The comparative characteristics of strength properties are given in Fig. 1, in which the curves are numbered in compliance with the above cement compositions.

The data given in Fig. 1 show that the Portland cement, modified with the dope based on the composite PC I-500-H + 0.075 \% (33.33\% NC : $22.67 \%$ STPP : $44 \%$ SHMP) provides a more substantial increase in the strength of cement stone. A level of the intensification of the gaining in strength is significant and by the seventh day of hardening 
it exceeded that of the strength values peculiar for the dope-free composition of 28 days old by more than $45 \%$.

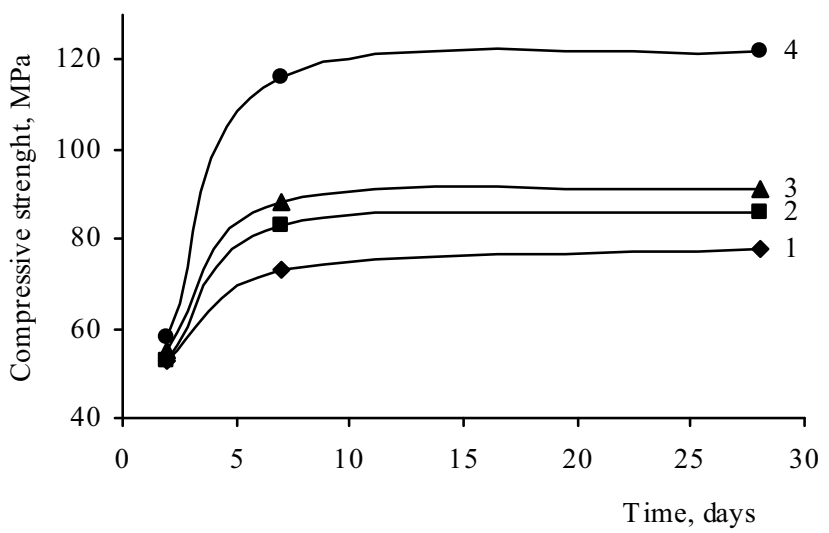

Fig. 1. Strength characteristics of tested cement compositions.

To define the phase composition of hydration products the specimens of hydrated dopefree Portland cement and those with an optimal composition of the composite dope were subjected to X-ray phase analysis. The X-ray phase analysis of tested specimens was done using the diffraction meter DRON-3M. The research data are given in the form of bar roentgenograms in Fig. 2.

The bar - roentgenograms given in Fig. 2 show the intensity of diffraction maxima for crystal compounds in hydration products and confirm the key conceptions of the mechanisms of a change in physical and mechanical characteristics of the tested objects. We believe that an increase in the strength of cement stone containing the introduced composite dope is caused by structural and phase changes during the process of hydration hardening.

The effect of strengthening in the presence of dope is provided due to a change in the character of the formation of crystal hydrate clusters and also due to qualitative and quantitative changes in the crystallization conditions of key phases. The dope contributes to a more complete hydration of basic clinker phases and an additional formation of tobermorite-like phases (interplane distances are 1.41, 1.268, 0.3076, $0.3001 \mathrm{~nm}$ ) of a different crystallinity and basicity and also to the formation of $\mathrm{AF}_{\mathrm{m}}$-phase (interplane distances correspond to those of ettringite that are identified by its strong peaks of 0.973 , 0.561 and $0.388 \mathrm{~nm}$ ).

A close connection exists between the structure strength and the character of it. However, these both properties are defined by the type and the volume of hydration products of the cementing material and different hydration phases possess different binding capacity. Tobermorite phases manifest the highest binding capacity in the cement paste. A.A. Govorov showed [10] that the stone strength is provided by new formations that are related to tobermorite and gyrolite groups and the strength of specimens based on the aluminum-substituted tobermorite is comparable with that of pure "xonotlite" structures. The formation of low-base calcium hydrosilicates with high specific surface results in a decrease of the efficient radius of pores and facilitates the formation of close pores that are not participating in the process of moisture transfer. All these changes decrease a degree of shrinkage and the gas and water permeability and result in an increase of the strength, deformability, crack resistance and the longevity that is provided due to a high content of 
calcium hydrosilicates of a reduced basicity that are capable of protecting hydroaluminate phases.

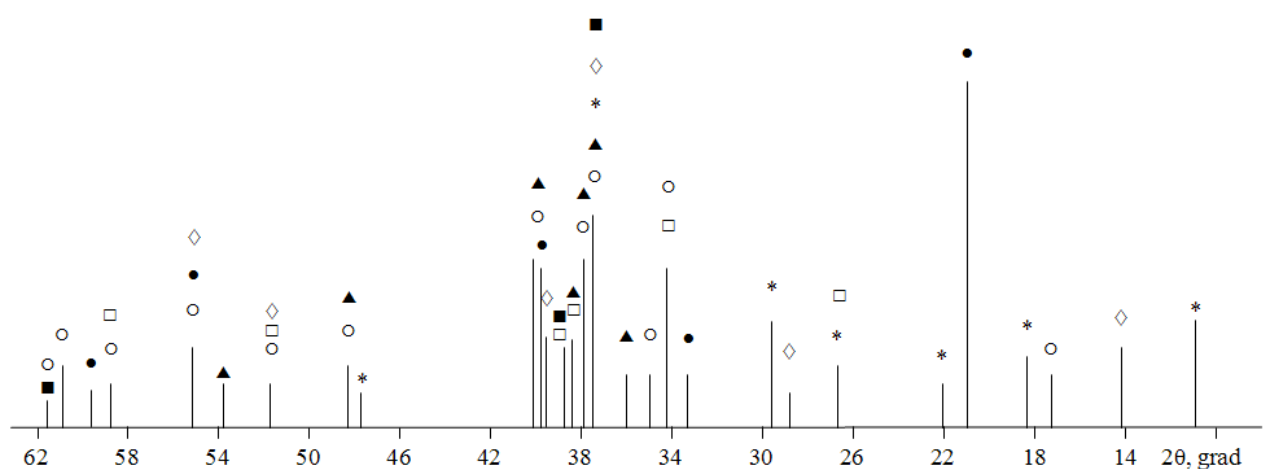

(a)

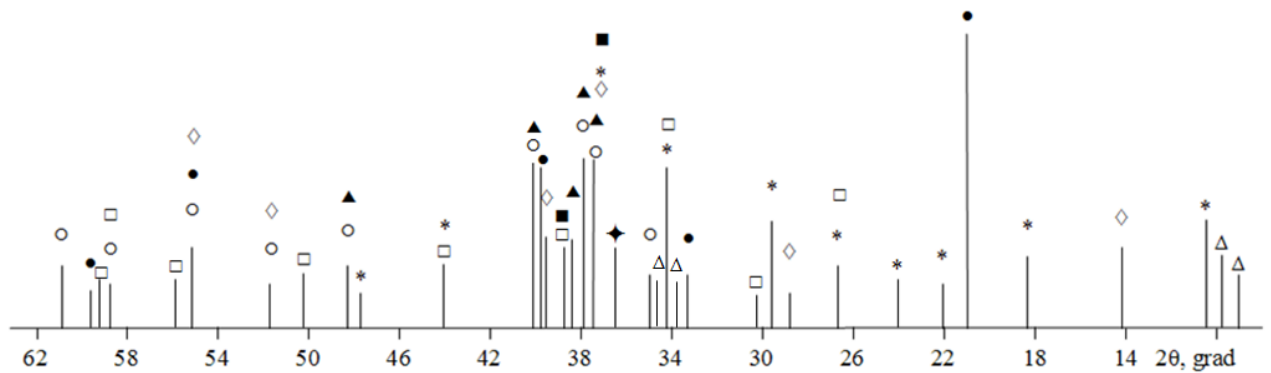

(b)

* $-\mathrm{Ca}_{6} \mathrm{Al}_{2}\left(\mathrm{SO}_{4}\right)_{3}(\mathrm{OH})_{12} \cdot 26 \mathrm{H}_{2} \mathrm{O}, \bullet-\mathrm{Ca}(\mathrm{OH})_{2}, \mathrm{O}-\mathrm{Ca}_{3} \mathrm{SiO}_{5}, \boldsymbol{\Delta}-\mathrm{Ca}_{2} \mathrm{SiO}_{4}$,

- $-\mathrm{Ca}_{3} \mathrm{Al}_{2} \mathrm{O}_{6}, \diamond-\mathrm{Ca}_{2}(\mathrm{Al}, \mathrm{Fe})_{2} \mathrm{O}_{5}, \square-\mathrm{CaCO}_{3}, \Delta$ - tobermorite-like phases, $-\mathrm{AF}_{\mathrm{m}}$-phase

Fig. 2. The bar - roentgenograms of hydrated cement compositions of 28 days old: (a) dope-free Portland-cement; (b) Portland cement with an optimal composition of the composite dope.

According to V.V. Timashev's data [10], the theoretical strength of low-base calcium hydrosilicates defined by their chemical composition and the structure of crystal lattice is actually two times higher than that of high-base calcium hydrosilicates. According to Y. Yambor data [10], the pore size distribution is an important factor that has a substantial influence on the material strength. In particular, it was shown that the binding compositions that contain hydration products of a similar type are characterized by such pore size distribution. Tobermorite and tobermorite phases contribute to the formation of the pores of an average size in the cement stone; the smallest of them have a size of 10 to $40 \mathrm{~nm}$ in contrast to other hydration products [10].

\section{Conclusions made using the research data and the prospects for further development in this area}

The main functional purpose of electrolyte dopes is considered to be an intensification of the gain in the concrete strength and the facilitation of cement hydration processes, producing a positive influence on the phase composition character of new hydrate formations and the pore space. An optimal relation of the dope ingredients that make up the cement-containing composition provides a considerable increase in the strength by the seventh day of hardening, more than $45 \%$ in comparison with the dope-free Portland cement at an age of 28 days. 
Application of the developed composite dope seems to be rather promising due to the modifying effect it has on the hydration hardening processes and a gain in the strength of hardening compositions and it can be also used for the production of materials that are characterized by a low emission of hazardous substances from the surface and have, as a result, an increased ecologic compatibility.

\section{References}

1. S.P. Sivkov, I.C. Kudysheva, Ammonia emission from cement concretes (19 Internationale Baustofftagung, Weimar, 2015)

2. V.N. Shimanov, Problem of emission of ammonia from concrete constructions (Access mode: http://www.science-education.ru/105-7025, 2015)

3. L.V. Predelskiy, O.E. Prihodchenko, Building ecology (Train aid, Rostov on Don, 2003)

4. Higuchi Takayuki, Method for electrochemical treatment of ready mixed concrete (Patent JP2004122620, 2004)

5. Pollak Vladimir, Chodak Ivan, Vtthod for the treatment of concrete (Patent SK151099, 2001)

6. Robert F. Rathbone, Thomas L. Robl. University of Kentucky Center for Applied Energy Research. Final Report. 63 (2001)

7. Z. Bai. Environment International 32, 303 (2006)

8. G.N. Shabanova, S.M. logvinkov, T.V. Shkolnikova, V.N. Shumejko. Collected scientific works of Ukrainian State University of Railway 162, 189 (2016)

9. V.S. Izotov, U.A. Sokolova, Chemical additions for modification of concrete (Monograph, Moscow, 2006)

10. Intercommunication of structure and properties of concrete (Access mode: http://stroiarchive.ru/shlakoschelochnoy-beton/1077-vzaimosvyaz-struktury-i-svoystvbetona.html, 2016) 